\title{
Enhanced suicide gene therapy using a tumor-specific promoter in combination with cisplatin
}

\author{
HONGYAN ZHANG**, ZHENG-KAI LIAO*, WEN-JIE SUN, CHENGHU HUANG, JIE XIONG, \\ FU-XIANG ZHOU, CONG-HUA XIE and YUN-FENG ZHOU
}

\begin{abstract}
Hubei Cancer Clinical Study Center, Hubei Key Laboratory of Tumor Biological Behaviors, Department of Radiochemotherapy, Zhongnan Hospital, Wuhan University, Wuhan 430071, Hubei, P.R. China
\end{abstract}

Received May 27, 2009; Accepted July 23, 2009

DOI: 10.3892/mmr_00000208

\begin{abstract}
Human telomerase reverse transcriptase (hTERT) promoter has been proposed in cancer-targeted gene therapy. However, this promoter has not been strong enough to achieve therapeutic levels of transgene expression. We favor the hypothesis that telomerase may participate in the process of DNA repair and that the up-regulation of hTERT promoter activity may be a reaction to DNA damage. In previous investigations, we tested an indirected-activator' strategy that utilizes radiation to increase the activity of the hTERT promoter. The purpose of the present study was to implement a strategy using cisplatin to enhance hTERT promoter activity. The results indicate that, in human uterine cervical cancer (HeLa) cells exposed to $5 \mu \mathrm{g} / \mathrm{ml}$ cisplatin, the hTERT promoter had 3.1-fold increased activity compared to untreated cells. In addition, the combination of cisplatin and hTERT promoter-mediated horseradish peroxidase/indole-3 -acetic acid gene-directed enzyme prodrug therapy induced cell cycle arrest at the $\mathrm{S}$ phase and apoptosis leading to a more significant reduction in cell viability. These findings suggest that hTERT promoter-mediated gene therapy is improved when combined with cisplatin.
\end{abstract}

\section{Introduction}

Selective gene therapy, which utilizes a tumor-specific promoter to control the transgene, represents a potent strategy in cancer treatment. Telomerase is a ribonucleoprotein complex that is activated in approximately $90 \%$ of cancers, but not in most normal cells (1). Human telomerase reverse transcriptase

Correspondence to: Dr Yun-Feng Zhou, Hubei Cancer Clinical Study Center, Hubei Key Laboratory of Tumor Biological Behaviors, Department of Radiochemotherapy, Zhongnan Hospital, Wuhan University, 169 Dong Hu Road, Wuhan 430071, Hubei, P.R. China E-mail: yfzhouwhu@gmail.com

*Contributed equally

Key words: hTERT promoter, horseradish peroxidase/indole-3acetic acid, suicide gene therapy, cisplatin, HeLa cells
(hTERT) is the catalytic subunit of telomerase, and determines telomerase activity (2). Thus, the hTERT promoter has been widely used in gene therapy targeting cancer cells $(3,4)$. However, the activity of the promoter may not be strong enough to induce the expression of a significant amount of the therapeutic gene product required to kill cancer cells $(5,6)$.

Radiotherapy is one of the major clinical treatment options for malignant tumors; however, cellular DNA damage responses are triggered when cells are exposed to irradiation. Data from our previous investigations suggested that telomerase plays a role in healing radiation-induced DNA damage (7). We also observed that radiation can be used to increase the activity of the hTERT promoter (8). Cisplatin is one of the most efficient chemotherapeutic agents in use, exerting its cytotoxic effect through the formation of intra-strand and inter-strand Pt-DNA cross-links. We favor the hypothesis that telomerase may participate in the process of DNA repair, and that the up-regulation of hTERT promoter activity may be a reaction to DNA damage. Thus, cisplatin may be used to enhance hTERT promoter activity. This is promising in cancer-targeted gene therapy.

The aim of the present study was to determine the potential of utilizing cisplatin to enhance hTERT promoter activity. The human uterine cervical cancer cell line HeLa was used, since the hTERT promoter has been applied to mediate tumorspecific expression in cervical cancer cells $(9,10)$. Cisplatin is the standard systemic chemotherapeutic agent for cervical cancer (11). The relationship between dosage of cisplatin and the induced activity of the hTERT promoter was examined. In addition, the horseradish peroxidase (HRP)/indole-3-acetic acid (IAA) gene-directed enzyme prodrug therapy (GDEPT) system was used to determine whether the combination of cisplatin and hTERT promoter-controlled gene therapy was effective as a cancer gene therapy. Proliferation rate and the apoptosis index were used to evaluate this strategy. Changes in cell cycle distribution were also investigated.

\section{Materials and methods}

Cell culture. The human uterine cervical cancer cell line HeLa (ATCC, CCL-2) was obtained from the China Center for Type Culture Collection. Cells were cultured in RPMI-1640 medium supplemented with 10\% calf serum (Gibco, USA) and maintained at $37^{\circ} \mathrm{C}$ in $5 \% \mathrm{CO}_{2}$ in a humidified incubator. 
Plasmid DNA and cell transfection. The core hTERT promoter $(-385 /+40$ bp) was kindly provided by Dr I. Horikawa (NIH/ NCI, USA) (12) and was cloned into the pGL3-Basic vector (Promega, USA) to generate phTERTp-Luc. For plasmid phTERTp, in which the hTERT promoter (hTERTp) does not control the expression of any gene, hTERTp replaced the CMV promoter in pcDNA3.1(-) (Invitrogen, USA). Plasmid pRK5-HRP was a kind gift from Dr D.F. Cutler (UCL, UK) (13). For phTERTp-HRP, the HRP cDNA was cloned into the phTERTp plasmid, driven by hTERTp. Cells were transfected using the Metafectene method (Biontex Laboratories $\mathrm{GmbH}$, Germany) according to the manufacturer's instructions.

Reporter gene assay. Plates (24-well) were seeded with $1 \times 10^{5}$ cells/well. At an optical confluency of $90-100 \%$, cells were cotransfected with $0.25 \mu \mathrm{g}$ of plasmid phTERTp-Luc or pGL3-Basic (negative control plasmid; Promega) and $0.25 \mu \mathrm{g}$ of pRL-TK (cotransfected standard plasmid; Promega) (Metafectene $2 \mu \mathrm{l}$ ) per well. Cells were exposed to 0,1,2 or $5 \mu \mathrm{g} / \mathrm{ml}$ cisplatin after transfection for $24 \mathrm{~h}$ and lysed for luciferase activity analysis using the Dual-luciferase reporter assay system (Promega) as indicated by the manufacturer. Internal normalization of transfection efficacy was performed using the value of Renilla luciferase (RL). The value of luciferase activity in negative control cells (cotransfected pGL3-Basic and pRL-TK) reflected the background, and was deducted.

Cell viability by MTT assay. The sensitivity of HeLa cells towards cisplatin was determined by the MTT assay as described previously (14). In brief, $3 \times 10^{4}$ cells/well were seeded in a 96-well plate. After $24 \mathrm{~h}$, the cells were transfected with plasmid phTERTp-HRP (DNA, Metafectene $0.1 \mu \mathrm{g}: 0.6 \mu \mathrm{l}$ ). After transfection for $24 \mathrm{~h}$, cisplatin at concentrations of 0,1 , 2 and $5 \mu \mathrm{g} / \mathrm{ml}$ was diluted with $200 \mu \mathrm{l}$ of medium and added to each well (6 parallel samples in each group). The cells were exposed to the drug for an additional $44 \mathrm{~h}$, then incubated for $4 \mathrm{~h}$ with $20 \mu \mathrm{l}$ of methylthiazolyltetrazolium (MTT; Sigma, USA) at a concentration of $5 \mathrm{~g} / 1$ at $37^{\circ} \mathrm{C}$ in a $5 \% \mathrm{CO}_{2}$ atmosphere. Subsequently, $150 \mu \mathrm{l}$ dimethyl sulphoxide was added to each well, and the cells were agitated for $10 \mathrm{~min}$. Cisplatin-treated cell viability was expressed as the percentage of cell proliferation of the control cells [(A492 treated cells/A492 control cells) x 100] after correction for background absorbance.

Analysis of apoptosis by flow cytometry. HeLa cells were washed twice with RPMI-1640 medium. Adherent cells were detached using $0.25 \%$ trypsin in $0.02 \%$ EDTA and pelleted by centrifugation at $800 \mathrm{rpm}$ for $5 \mathrm{~min}$. The cells $\left(1 \times 10^{5}\right)$ were washed twice in PBS at $4^{\circ} \mathrm{C}$ and resuspended in $100 \mu \mathrm{l}$ of binding buffer containing Annexin V/FITC (5 $\mu \mathrm{l})$ and propidium iodide (PI) $(10 \mu \mathrm{l}, 20 \mu \mathrm{g} / \mathrm{ml})$ for $15 \mathrm{~min}$ in the dark. With the addition of $0.4 \mathrm{ml}$ PBS, stained cells were analyzed for apoptosis by collecting 10,000 counts using the Cytomics ${ }^{\mathrm{TM}}$ FC500 Flow Cytometer (Beckman Coulter, USA).

Cell cycle analysis by flow cytometry. Cell cycle phases (G0/ $\mathrm{G} 1, \mathrm{~S}$ or $\mathrm{G} 2 / \mathrm{M}$ ) were characterized according to DNA content. The fluorescent dye PI binds with DNA strongly at a ratio of 1:1; hence, the DNA contents of the cell cycle phases were reflected by varying PI fluorescence intensities. HeLa cells

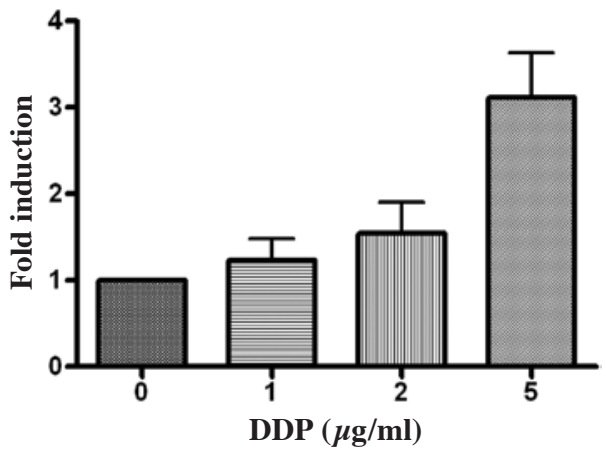

Figure 1. Effect of cisplatin (DDP) on exogenous hTERT promoter activity in HeLa cells. hTERT promoter activity after exposure to cisplatin in HeLa cells was evaluated by luciferase activity assay. Cells were treated with increasing concentrations of cisplatin and collected $24 \mathrm{~h}$ after exposure. Exogenous hTERT promoter activity was expressed as the fold induction relative to that of cells that received $0 \mu \mathrm{g} / \mathrm{ml}$ cisplatin treatment. Data are expressed as the means $\pm \mathrm{SD}$ of three experiments performed in triplicate.

were washed twice with RPMI-1640 medium, and adherent cells were detached using $0.25 \%$ trypsin in $0.02 \%$ EDTA and pelleted by centrifugation at $800 \mathrm{rpm}$ for $5 \mathrm{~min}$. The cells $\left(1 \times 10^{5}\right)$ were washed twice in PBS at $4^{\circ} \mathrm{C}$ and fixed in $70 \%$ ice-cold $\left(4^{\circ} \mathrm{C}\right)$ ethanol. Cell pellets were resuspended in $1 \mathrm{mg} /$ $\mathrm{ml}$ RNase solution (Sigma, USA) for $30 \mathrm{~min}$ at $37^{\circ} \mathrm{C}$, and then in $0.1 \mathrm{mg} / \mathrm{ml} \mathrm{PI}$ solution (Sigma) for $30 \mathrm{~min}$ at $4^{\circ} \mathrm{C}$ in the dark. Cell cycle analysis was performed on the Cytomics ${ }^{\mathrm{TM}}$ FC500 flow cytometer.

Statistical analysis. Results are expressed as the mean \pm SD. Differences between groups were analyzed by the one-way ANOVA test. Data were analyzed using SPSS13.0 software (SPSS Inc., USA). The differences were considered statistically significant at $\mathrm{P}<0.05$.

\section{Results}

Effect of cisplatin on the activity of the hTERT promoter in HeLa cells. In order to establish whether exogenous hTERT promoter activity was modulated by cisplatin, firefly luciferase was used as the reporter gene to determine the instantaneous activation of the hTERT promoter after cisplatin exposure. RL was used for internal normalization, and pGL3-Basic was used as a negative control and background value. Transfected HeLa cells were treated for $24 \mathrm{~h}$ with increasing concentrations of cisplatin, ranging from 0 to $5 \mu \mathrm{g} / \mathrm{l}$. As shown in Fig. 1, induced activity of the hTERT promoter was expressed in a dose-dependent manner. The peak of hTERT promoter activity (3.11 \pm 1.03 -fold increase) was detected in cells treated with $5 \mu \mathrm{g} /$ $\mathrm{ml}$ cisplatin (Fig. 1). In order to verify the induction of hTERT promoter by cisplatin, $5 \mu \mathrm{g} / \mathrm{ml}$ of cisplatin was adopted for the following experiments.

Effect of cisplatin on HeLa cell proliferation. The cytotoxicity of cisplatin to HeLa cervical cancer cells was investigated after an incubation period of $48 \mathrm{~h}$ using the colorimetric MTT assay. As shown in Fig. 2, HeLa cell proliferation was inhibited in a dose-dependent manner in response to increasing concentrations of cisplatin $(0-5 \mu \mathrm{g} / \mathrm{ml})$. Inhibition of $49.61 \pm 8.94 \%$ was 


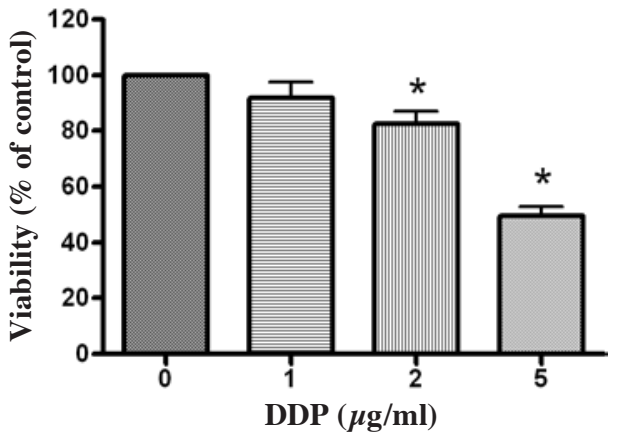

Figure 2. Cytotoxicity of various concentrations of cisplatin (DDP) on HeLa cells according to the MTT assay. The cell viability of cells at $0 \mu \mathrm{g} / \mathrm{ml}$ was set as $100 \%$ and used for comparison. As the concentration of cisplatin increased, cell viability decreased. Data are expressed as the means \pm SD of three independent experiments $\left({ }^{*} \mathrm{P}<0.01\right)$.

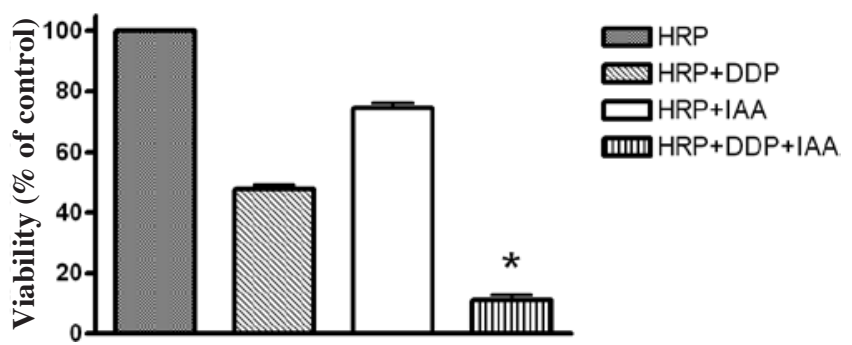

Figure 3. Cytotoxic effects on HeLa cells. Cell viability was measured by MTT assay (see Materials and methods). Combining phTERTp-HRP/IAA with cisplatin resulted in the lowest viability. The cell viability of the cells was compared to that of the HRP+DDP+IAA group. Data are expressed as the means $\pm \mathrm{SD}$ of three independent experiments * Statistically significant difference $(\mathrm{P}<0.01)$

obtained with a cisplatin concentration of $5 \mu \mathrm{g} / \mathrm{ml}$. In contrast, no obvious cytotoxicity to HeLa cells was observed after incubation with $1 \mu \mathrm{g} / \mathrm{ml}$ of cisplatin, as verified by the MTT assay (Fig. 2).

Effect of combining phTERTp-HRP/IAA with cisplatin on cell viability. The efficacy of hTERT promoter induction was investigated after the HeLa cells were exposed to $5 \mu \mathrm{g} / \mathrm{ml}$ of cisplatin (Fig. 1). We hypothesized that cisplatin could be used to improve the efficiency of hTERT promoter-mediated gene therapy. To evaluate the application of this strategy, phTERTp-HRP plasmids were transiently transfected into HeLa cells. Twenty four hours after transfection, the cells were divided into four groups according to treatment: a) HRP (PBS); b) HRP+cisplatin (DDP) (5 $\mu \mathrm{g} / \mathrm{ml}$ DDP); c) HRP+IAA (0.5 mmol/l IAA); and d) HRP+DDP+IAA (5 $\mu \mathrm{g} / \mathrm{ml}$ DDP and $0.5 \mathrm{mmol} / \mathrm{l}$ IAA).

The cytotoxic effects of combining phTERTp-HRP/IAA with cisplatin on HeLa cells were measured following treatment using the MTT assay (Fig. 3). Inhibition of $25.34 \pm 3.40 \%$ was obtained in transfected HeLa cells after incubation with 0.5 mM IAA, while $47.80 \pm 3.46 \%$ of the cells survived exposure to $5 \mu \mathrm{g} / \mathrm{ml}$ cisplatin. Conversely, when HRP/IAA controlled by the hTERT promoter was combined with cisplatin, only $11.05 \pm 3.67 \%$ of the HeLa cells survived IAA incubation, indicating the synergistic effect of this combination strategy.
A
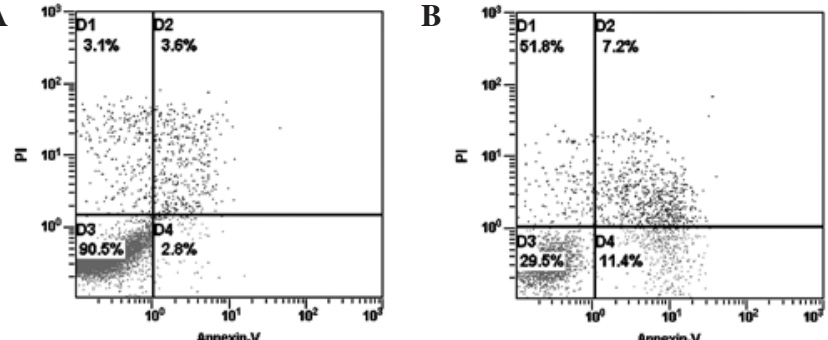

C

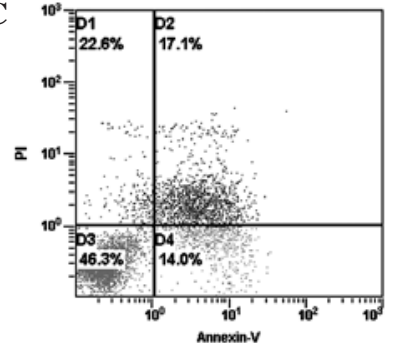

D

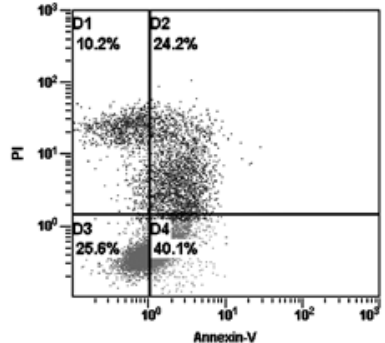

$\mathbf{E}$

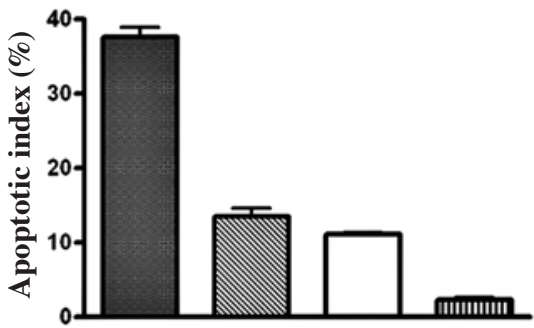

$\square \mathrm{HRP}+\mathrm{DDP}+\mathrm{IAA}$

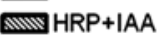

$\longrightarrow H R P+D D P$ IIIIII)HRP

Figure 4. Combination of phTERTp-HRP/IAA with cisplatin (DDP) in HeLa cells. During the early stages of cell apoptosis, phosphatidylserine (PS) was translocated from the inner layer of the plasma membrane to the outer layer, resulting in irreversible programmed cell death (apoptosis). FITC-labeled Annexin V bound to PS and was detected by flow cytometry, indicating the initiation of cell apoptosis. The fluorescent dye propidium iodide (PI) was used to stain the cytoplasm and nucleus via pores formed on the cell membrane, indicating late-stage apoptosis or cell death. FITC+PI- cells were determined to be undergoing the early stages of apoptosis, and FITC $+\mathrm{PI}^{+}$cells, the late stages of apoptosis or cell death due to other reasons, such as necrosis. Only FITC+PI- cells were used to determine early apoptosis in the analysis. The apoptotic index is presented as the percentage of apoptotic cells versus total cells. (A) HRP (PBS); (B) HRP+DDP (5 $\mu \mathrm{g} / \mathrm{ml}$ DDP); (C) HRP+IAA (0.5 mmol/1 IAA); (D) HRP+DDP+IAA $(5 \mu \mathrm{g} / \mathrm{ml}$ DDP and $0.5 \mathrm{mmol} / 1$ IAA). (E) HeLa cells were treated with phTERTp-HRP/IAA and cisplatin, and showed a greatly increased rate of early apoptosis compared to the other groups ( $\mathrm{P}<0.01$ for all groups).

Effect of combining phTERTp-HRP/IAA with cisplatin on cell apoptosis and the cell cycle. The significant growth inhibitory activity of combining phTERTp-HRP/IAA with cisplatin led us to investigate whether part of this effect was the result of changes in programmed cell death induction and the cell cycle.

As shown in Fig. 4, apoptosis analysis indicated that HeLa cells treated with a combination of phTERTp-HRP/IAA and cisplatin underwent marked apoptosis with an early apoptotic rate of $39.82 \pm 2.61 \%, 14.2$-fold higher than that of the control cells. A 4.1-fold increase in the apoptosis of cells treated with cisplatin alone and a 5.0-fold increase in those treated with IAA alone was estimated and compared to the control cells, as assessed by the early apoptotic rate.

Cell cycle analysis revealed that the combination treatment provoked an inhibition of $\mathrm{S}$ phase cell cycle progression (Table I). The combination of phTERTp-HRP/IAA and cisplatin induced an increase in the number of cells in the $S$ phase 
Table I. Cell cycle phase distribution of HeLa cells after a 48-h treatment.

\begin{tabular}{lccc}
\hline Group & G0/G1 $(\%)$ & $\mathrm{S}(\%)$ & $\mathrm{G} 2 / \mathrm{M}(\%)$ \\
\hline Control & $62.32 \pm 0.078$ & $19.55 \pm 0.300$ & $18.12 \pm 0.244$ \\
DDP & $52.45 \pm 2.778$ & $30.10 \pm 1.136$ & $17.45 \pm 2.139$ \\
phTERTp-HRP/IAA & $64.14 \pm 2.288$ & $23.01 \pm 1.710$ & $12.85 \pm 1.433$ \\
Combination & $52.87 \pm 1.754$ & $32.69 \pm 3.027$ & $14.44 \pm 1.370$ \\
\hline
\end{tabular}

Cells exposed to the combination of phTERTp-HRP/IAA and cisplatin exhibited an increase during the S phase and a decrease during the G0/G1 and $\mathrm{G} 2 / \mathrm{M}$ phases.

$(32.69 \pm 3.03 \%)$ as compared to the control cells $(19.55 \pm 0.30 \%)$. This phase change was confirmed by a reciprocal observation that combination treatment resulted in a significant decrease in the number of cells in the G0/G1 phase $(52.86 \pm 1.75 \%)$ and $\mathrm{G} 2 / \mathrm{M}$ phase $(14.44 \pm 1.37 \%)$ compared to the control cells $(62.32 \pm 0.08$ and $18.12 \pm 0.24 \%$, respectively).

\section{Discussion}

Cervical cancer is the seventh most frequently occurring type of cancer worldwide, with a global incidence in 2002 of 493,243 cases and a mortality rate of 273,505 deaths per year (15). In developing countries, cervical cancer is the second leading cause of cancer mortality in women (15). Effective cytotoxic treatment options for advanced cervical cancer are exceedingly limited (16), and the survival rate of patients with cervical cancer has not improved substantially over the past 25 years (17). Cisplatin is regarded as the most active agent in carcinoma of the cervix, and cisplatin-based combination chemotherapy is most commonly applied, resulting in response rates of 7-78\% and overall survival of less than 25 months (11). New treatment strategies are therefore necessary. Cancer gene therapy is an interesting approach for cervical cancer patients, as the specific delivery of DNA vector to cancer cells by local injection is feasible.

One limitation of cancer gene therapy is its toxicity to normal cells. Targeted gene therapy exploits differences between malignant and normal tissue cells in order to preferentially express therapeutic genes in tumor cells. Telomerase is a ribonucleoprotein complex activated in approximately $90 \%$ of cancers, but not in most normal cells (1). Human telomerase consists of a catalytic protein subunit (hTERT) and a RNA subunit (hTR). The transcriptional regulation of hTERT largely controls telomerase activity (2). hTERT promoter with activation only in cells with active telomerase has been widely used in gene therapy for targeting cancer cells $(3,4)$. However, hTERT promoter may not be strong enough to achieve therapeutic levels of transgene expression $(5,6)$.

Telomerase activation is a relatively early-stage event in cervical carcinogenesis (18), and it may be possible to use the hTERT promoter to restrict transgene expression to cervical cancer cells. On this basis, researchers have developed novel strategies mediated by the hTERT gene promoter to achieve tumor-selective killing ability for cervical cancer $(9,10)$. However, to maximize the therapeutic efficiency of this strategy, it is necessary to enhance the activity of the
hTERT promoter. We previously demonstrated that telomerase participates in the process of DNA repair, and that the upregulation of telomerase activity may be a reaction to DNA damage induced by irradiation (7). Thus, the hTERT promoter may be indirectly enhanced in response to DNA-damaging agents. In our previous studies, we found that the activities of exogenous and endogenous hTERT promoter in HEp-2 cells can be induced by irradiation. This 'indirected-activator' strategy may be capable of amplifying the targeted killing effect of hTERT promoter-mediated gene therapy in vitro and in vivo $(8,19)$.

Cisplatin is one of the most widely-used DNA-damaging agents in the treatment of cancer. In this study, cisplatin not only killed tumor cells by damaging DNA, but also up-regulated the activity of the hTERT promoter. Using a dual-luciferase reporter assay, we observed that the hTERT promoter was maximally enhanced by $5 \mu \mathrm{g} / \mathrm{ml}$ cisplatin compared to other doses. However, the published data on the relationship between the effects of cisplatin and changes in telomerase activity are conflicting (20). In this study, low-dose $(0-2 \mu \mathrm{g} / \mathrm{ml})$ cisplatin exposure resulted in little enhancement of hTERT promoter activity. This may be due to the minimal DNA damage it effected. In contrast, high dose cisplatin treatment resulted in marked cancer cell killing, and telomerase activity was decreased in dying cells (21). Since the precise mechanism underlying the observed changes in hTERT promoter activity in cancer cells is unclear, detailed investigations are needed. Here, cell cycle arrest at the S phase was demonstrated in HeLa cells in response to cisplatin, in accordance with the results of other studies $(22,23)$. It is known that telomerase synthesizes telomeres during the $\mathrm{S}$ phase, and that telomerase activity markedly increases during this phase of the cell cycle (24). Furthermore, the hTERT promoter mediated gene therapy in a strictly cancer- and S-phase-specific manner (25). Thus, we hypothesized that the up-regulation of hTERT promoter activity may also be the result of cell cycle arrest in the $\mathrm{S}$ phase induced by ciplatin.

Gene-directed enzyme prodrug therapy (GDEPT) is a form of tumor-targeted chemotherapy that has entered several clinical trials. The HRP/IAA system, a novel GDEPT system, has shown great efficacy in killing tumor cells, exhibiting a strong bystander effect and significant synergism with radiation, as well as greater cytotoxicity to tumors than the well-known HSVtk/GCV system (26,27). HRP/IAA involves the delivery of a specific enzyme (HRP) that converts non-cytotoxic IAA into cytotoxic metabolites. When the HRP gene is targeted to 
tumor cells, only the tumor and surrounding cells are affected by the cytotoxic metabolites, generating a bystander effect (28). Crucially, under normal conditions, HRP is absent from mammalian cells. IAA is a poor substrate for mammalian peroxidases, thus avoiding the possibility of systemic toxicity $(29,30)$. Tumor-specific expression of the HRP gene is crucial for minimizing the side effects of treatment. In this study, in order to improve hTERT promoter-mediated GDEPT, phTERTp-HRP/IAA targeted gene therapy was combined with cisplatin exposure. The results revealed a synergistic inhibition of proliferation. Although necrosis, DNA fragmentation, chromatin condensation and apoptosis are involved in the cytotoxic effect of HRP/IAA GDEPT $(26,31)$, apoptosis is the most important mechanism of cytotoxicity (32). Combination strategy enhanced apoptosis in HeLa cells and induced cell cycle arrest at the $\mathrm{S}$ phase. This is likely to contribute to the inhibition of proliferation in HeLa cells.

In this study, cisplatin-induced DNA damage was used as a therapeutic tool and as an activator to enhance the activity of the hTERT promoter. The results provide further support for the indirected-activator' strategy, by which DNA damage can be used to enhance the hTERT promoter in targeted gene therapy. This hypothesis may provide a novel explanation for the satisfactory results obtained by other researchers combining cisplatin with gene therapy using the hTERT promoter (9,33-35).

Cisplatin is one of the most effective chemotherapeutic agents, and has been successfully used to treat tumors of the head, neck, lungs and genitourinary tract (36). The hTERT promoter has been widely used in gene therapy for targeted cancer cells $(3,4)$. Combined with cisplatin, hTERT promoter-mediated cancer-targeted gene therapy approaches may achieve a more satisfactory curative effect. The results presented here demonstrate for the first time that cisplatin enhances the activity of the hTERT promoter in human uterine cervical cancer cells. Our data support the hypothesis that the tumor-targeted hTERT promoter drives the transcription of transgenes in response to DNA-damaging agents.

\section{Acknowledgements}

This study was supported by the National Science Foundation of China (NSFC) (grant no. 30672438) and the Natural Science Foundation of Hubei Province (grant no. 2006ABC009).

\section{References}

1. Kim NW, Piatyszek MA, Prowse KR, et al: Specific association of human telomerase activity with immortal cells and cancer. Science 266: 2011-2015, 1994.

2. Cong YS, Wright WE and Shay JW: Human telomerase and its regulation. Microbiol Mol Biol Rev 66: 407-425, 2002.

3. Fakhoury J, Nimmo GA and Autexier C: Harnessing telomerase in cancer therapeutics. Anticancer Agents Med Chem 7: 475-483, 2007.

4. Wirth T, Kuhnel F and Kubicka S: Telomerase-dependent gene therapy. Curr Mol Med 5: 243-251, 2005.

5. Gu J and Fang B: Telomerase promoter-driven cancer gene therapy. Cancer Biol Ther 2: S64-S70, 2003.

6. Keith WN, Bilsland A, Hardie M and Evans TR: Drug insight: Cancer cell immortality - telomerase as a target for novel cancer gene therapies. Nat Clin Pract Oncol 1: 88-96, 2004.

7. Zhou FX, Liao ZK, Dai J, et al: Radiosensitization effect of zidovudine on human malignant glioma cells. Biochem Biophys Res Commun 354: 351-356, 2007.
8. Liao ZK, Zhou FX, Luo ZG, et al: Radio-activation of hTERT promoter in larynx squamous carcinoma cells: an 'indirectedactivator' strategy in radio-gene-therapy. Oncol Rep 19: 281-286, 2008.

9. Nakamura M, Kyo S, Kanaya T, et al: hTERT-promoter-based tumor-specific expression of MCP-1 effectively sensitizes cervical cancer cells to a low dose of cisplatin. Cancer Gene Ther 11: 1-7, 2004

10. Lanson NA Jr., Friedlander PL, Schwarzenberger P, Kolls JK and Wang G: Replication of an adenoviral vector controlled by the human telomerase reverse transcriptase promoter causes tumorselective tumor lysis. Cancer Res 63: 7936-7941, 2003.

11. Pectasides D, Kamposioras K, Papaxoinis G and Pectasides E: Chemotherapy for recurrent cervical cancer. Cancer Treat Rev 34: 603-613, 2008.

12. Horikawa I, Cable PL, Mazur SJ, Appella E, Afshari CA and Barrett JC: Downstream E-box-mediated regulation of the human telomerase reverse transcriptase (hTERT) gene transcription: evidence for an endogenous mechanism of transcriptional repression. Mol Biol Cell 13: 2585-2597, 2002.

13. Connolly CN, Futter CE, Gibson A, Hopkins CR and Cutler DF: Transport into and out of the Golgi complex studied by transfecting cells with cDNAs encoding horseradish peroxidase. J Cell Biol 127: 641-652, 1994.

14. Ayed-Boussema I, Bouaziz C, Rjiba K, et al: The mycotoxin Zearalenone induces apoptosis in human hepatocytes (HepG2) via p53-dependent mitochondrial signaling pathway. Toxicol In Vitro 22: 1671-1680, 2008.

15. Parkin DM, Bray F, Ferlay J and Pisani P: Global cancer statistics, 2002. CA Cancer J Clin 55: 74-108, 2005.

16. Del Campo JM, Prat A, Gil-Moreno A, Perez J and Parera M Update on novel therapeutic agents for cervical cancer. Gynecol Oncol 110: S72-S76, 2008.

17. Jemal A, Siegel R, Ward E, et al: Cancer statistics, 2008. CA Cancer J Clin 58: 71-96, 2008.

18. Rosa MI, Medeiros LR, Bozzetti MC, et al: Accuracy of telomerase in cervical lesions: a systematic review. Int J Gynecol Cancer 17: 1205-1214, 2007.

19. Liao Z, Huang C, Zhou F, et al: Radiation enhances suicide gene therapy in radioresistant laryngeal squamous cell carcinoma via activation of a tumor-specific promoter. Cancer Lett: (In press).

20. Jeyapalan JC, Saretzki G, Leake A, Tilby MJ and von Zglinicki T: Tumour-cell apoptosis after cisplatin treatment is not telomere dependent. Int J Cancer 118: 2727-2734, 2006.

21. Blasiak J, Kadlubek M, Kowalik J, Romanowicz-Makowska H and Pertynski T: Inhibition of telomerase activity in endometrial cancer cells by selenium-cisplatin conjugate despite suppression of its DNA-damaging activity by sodium ascorbate. Teratog Carcinog Mutagen 22: 73-82, 2002.

22. Lee BJ, Chon KM, Kim YS, et al: Effects of cisplatin, 5-fluorouracil, and radiation on cell cycle regulation and apoptosis in the hypopharyngeal carcinoma cell line. Chemotherapy 51: 103-110, 2005.

23. Bannon JH, Fichtner I, O'Neill A, et al: Substituted titanocenes induce caspase-dependent apoptosis in human epidermoid carcinoma cells in vitro and exhibit antitumour activity in vivo. Br J Cancer 97: 1234-1241, 2007.

24. Zhu X, Kumar R, Mandal M, et al: Cell cycle-dependent modulation of telomerase activity in tumor cells. Proc Natl Acad Sci USA 93: 6091-6095, 1996

25. Murofushi Y, Nagano S, Kamizono J, et al: Cell cycle-specific changes in hTERT promoter activity in normal and cancerous cells in adenoviral gene therapy: A promising implication of telomerase-dependent targeted cancer gene therapy. Int J Oncol 29: 681-688, 2006.

26. Greco O, Dachs GU, Tozer GM and Kanthou C: Mechanisms of cytotoxicity induced by horseradish peroxidase/indole-3-acetic acid gene therapy. J Cell Biochem 87: 221-232, 2002.

27. Greco O, Folkes LK, Wardman P, Tozer GM and Dachs GU: Development of a novel enzyme/prodrug combination for gene therapy of cancer: horseradish peroxidase/indole-3-acetic acid. Cancer Gene Ther 7: 1414-1420,2000.

28. Tupper J, Greco O, Tozer GM and Dachs GU: Analysis of the horseradish peroxidase/indole-3-acetic acid combination in a threedimensional tumor model. Cancer Gene Ther 11: 508-513, 2004.

29. Kobayashi S, Sugioka K, Nakano M, Takyu C, Yamagishi A and Inaba $\mathrm{H}$ : Excitation of indole acetate in myeloperoxidase- $\mathrm{H} 2 \mathrm{O} 2$ system: possible formation of indole acetate cation radical. Biochem Biophys Res Commun 93: 967-973, 1980.

30. Folkes LK, Candeias LPand Wardman P: Toward targeted 'oxidation therapy' of cancer: peroxidase-catalysed cytotoxicity of indole-3acetic acids. Int J Radiat Oncol Biol Phys 42: 917-920, 1998. 
31. De Melo MP, De Lima TM, Pithon-Curi TC and Curi R: The mechanism of indole acetic acid cytotoxicity. Toxicol Lett 148: 103-111, 2004.

32. Kim DS, Jeon SE, Jeong YM, Kim SY, Kwon SB and Park KC: Hydrogen peroxide is a mediator of indole-3-acetic acid/horseradish peroxidase-induced apoptosis. FEBS Lett 580: 1439-1446, 2006.

33. Takeuchi H, Kanzawa T, Kondo Y, et al: Combination of caspase transfer using the human telomerase reverse transcriptase promoter and conventional therapies for malignant glioma cells. Int J Oncol 25: 57-63, 2004.

34. Li B, Fan J, Liu X, et al: Suppression of colorectal tumor growth by regulated survivin targeting. J Mol Med 84: 1077-1086, 2006.
35. Toyota H, Kondo S, Kyo S and Mizuguchi J: Enforced expression of a truncated form of Bax-alpha (tBax) driven by human telomerase reverse transcriptase (hTERT) promoter sensitizes tumor cells to chemotherapeutic agents or tumor necrosis factor-related apoptosis-inducing ligand (TRAIL). Anticancer Res 26: 99-105, 2006.

36. Cohen SM and Lippard SJ: Cisplatin: from DNA damage to cancer chemotherapy. Prog Nucleic Acid Res Mol Biol 67: 93-130, 2001. 